\title{
BLOCKCHAIN"' HEALTHCARE
}

DISCUSSION

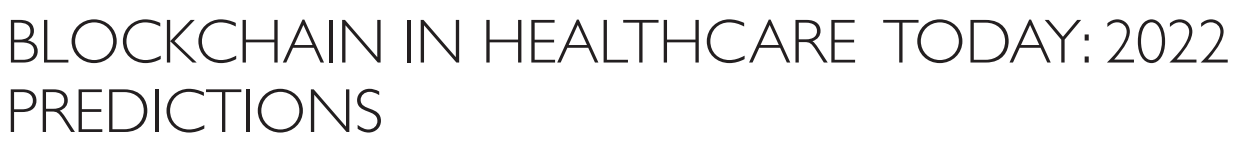

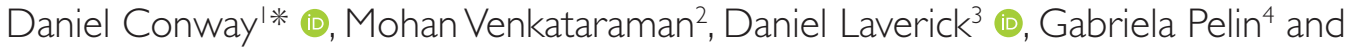 \\ Anton Hasselgren ${ }^{5}$ (D)
}

'Associate Director, Blockchain Center of Excellence, Clinical Professor of Information Systems, University of Arkansas, USA; ${ }^{2}$ ChiefTechnology Officer, Chainyard, a Business Unit of IT People Corp, USA; ${ }^{3}$ Vice President of Digital \& Data Solutions, Mobile, Alabama; ${ }^{4}$ Chief Innovation Officer, Avaneer Health, USA; ${ }^{5}$ Department of Neuromedicine and Movement Science, Faculty of Medicine and Health Sciences, Norwegian University of Science and Technology, Trondheim, Norway

Abstract

Each year, Blockchain and Healthcare Today reaches out to journal board members, annual ConV2X Symposium speakers, and ecosystem subject matter experts to share their near-term views and perspectives for blockchain technology advances in healthcare. This article presents insights into where authors anticipate market opportunities and where gaps exist that should be addressed for regional and global collaboration, governance, and efficiency for the year 2022.

Keywords: Supply chain; pharmaceutical manufacturers; chain of custody; smart contracts; cryptocurrency; health information exchanges; blockchain in healthcare; decentralized ledgers; fast healthcare interoperability resources (FHIR); id keychain; master index

Received: 06 December 2021; Revised: 21 December 2021; Accepted: 21 December 202 I; Published: 31 January 2022

$\mathrm{T}$ hat great philosopher, Yogi Berra, once said, 'It's tough to make predictions, especially about the future'. Despite such difficulty and despite plans often referred to as 'worthless', planning is everything.

Predictions are generally done in several ways. The most common approach is to project meaningful patterns of the past into the future - a momentum approach. We look for trends and seasonality over the past several years and project them forward into 2022. A contrarian approach might suggest a regression to the mean, where the mean itself is projected, but that recent deviations from that mean are likely to reverse. It is, after all, the role of management to make sure the future does not look like the past.

We also predict by looking for similarity and suggesting that similar events will reoccur. For example, those who liked these three books will also like this fourth book. Many technology trends, such as sales of $5 \mathrm{G}$ equipment, are predicted by looking at trends of similar products and suggesting a similar adoption maturity.
In the case of blockchain, we have a technology that is what we refer to as a foundational technology. Blockchain is not an improvement on an existing technology. Thus, we are left without a suitable comparison. Blockchain is the technology layer of a digital ecosystem, and we have not had that before.

It is difficult to predict. But, it is not over.

In the short term, we tend to consider other approaches, such as watching patent applications, paying membership trends in industry consortium, transaction volume patterns, other meta-data, and of course - we follow the money. Patent activity suggests that blockchain will soon be involved in PoS (point of sale) transactions. This is likely to occur when regulators formalize their approach to the new asset class of cryptocurrency. Large retailers and supply chains are building the transaction infrastructures to support this eventuality today.

Consortium membership suggests a strong move away from private permissioned blockchains toward confidentiality over public blockchains and toward confidential swap automated market makers. 
And the money following? While the transaction volume is increasing dramatically, within our scope, there are several efforts that are attracting attention.

- One such effort is that of the sovereign identity. Identity was not part of the original internet design, and we have struggled with it ever since. There is significant effort at standardizing this layer.

- A second effort to note is the increased interest in IoT (Internet of Things) expansion, where blockchain serves as the bookends that define where the ecosystem data should reside. Blockchains should contain minimal information, and internets of blockchains (such as Cosmos) enable a fairly robust separation of roles architectures.

- Another effort involves distributed replicated file systems, where the blockchain serves as an information repository and is resistant to single points of failure. This eliminates the need for business continuity and disaster recovery IT issues that most in the medical world hope go away anyway.

- Finally, with the expansion of $5 \mathrm{G}$ networks and some standardization of protocols, we see significant resources coming into the augmented reality and metaverse space. This should continue to reduce the location obstacles for providing healthcare services, globally.

Cars go fast only if they have good braking systems. The blockchain community welcomes a clear regulatory structure, but currently, that effort trails innovation. We would like to predict a change in the regulatory response, but prediction confidence is lacking.

In the following, we share insights that can be useful in predicting blockchain in healthcare for 2022 .

\section{Mohan Venkataraman, Chief Technology Officer, Chainyard, a Business Unit of IT People Corp, USA}

Technology plays a critical role in the healthcare industry, including drug discovery, clinical research, pharmaceutical manufacturing and distribution, patient care, and health plan administration. Hospitals, laboratories, and clinics use intelligent devices powered by sensors and AI (artificial intelligence) to detect, diagnose, and administer care. The industry is heavily regulated, and compliance requirements are quite complex. The most notable one is HIPAA (Health Insurance Portability and Accountability Act of 1996) whose primary focus is privacy of patient information and individual consent prior to sharing data. Taking a drug to market requires pharmaceutical companies to track and trace every step of the process and provide evidence to regulatory agencies until the drug gets final approval.
Medical facilities must maintain 'Chain of Custody' to prove that samples, specimens, and other physical materials collected have been properly handled from origination to final disposal. Patients must trust data that intelligent medical devices collect, and the associated recommendations made by $\mathrm{AI}$ and ML (machine learning) algorithms.

Blockchain will play a critical role in transforming healthcare in conjunction with peer technologies, such as AI, ML, and IOT. We are only at the beginning of this new revolution, as the technologies are still maturing. In the future, we see intelligent edge networks communication with the core, and blockchains will evolve into network of networks. Based on the inquiries received from healthcare-related companies, Chainyard sees significant applications of those technologies in the next 3 years. The blockchain will address the three main areas of concern (i.e. trust, transparency, and privacy). The coronavirus disease 2019 (COVID-19) pandemic has accelerated adoption of blockchain and peer technologies.

Fake PPEs (personal protective equipment) and sanitary products have necessitated the need for Supplier Qualification and Supply Chain Diversity. Chainyard's 'Trust Your Supplier' network has been the key player in this space and is expected to see growth. The second use-case stems from the fact that people are very concerned about the ingredients that go into the production of drugs, vitamins, and vaccines. Hence, drug approvals, manufacture, distribution of pharmaceuticals, and the origin and sourcing of the ingredients that compose the final product will all see an increase in the adoption of blockchain for 'track and trace' and 'regulatory reporting'. Third, the rise of COVID Passports will evolve into a broader Personal Health Wallet that will enable individuals to carry their records in tamper proof cryptographically secured digital wallets and enable cryptographic consent for sharing personal data. Fourth, Health Insurance companies will form consortiums to improve trust and enable more transparency in benefit administration and claims process - especially in lowering prescription costs. Lastly, intelligent devices at the edge that combine sensor technology and AI will have to be auditable, and hence, new 'Oracles' will emerge to record proofs of data origin and AIML (Artificial Intelligence Markup Language) algorithm signatures that processed the data to deliver insights and outcomes.

\section{Daniel Laverick, Vice President of Digital \& Data Solutions, Zuellig Pharma, Singapore}

Due to the COVID-19 pandemic restricting travel and face-to-face contact, the adoption of telehealth programs has risen astronomically out of necessity.

McKinsey reports (1) telehealth utilization stabilizing at levels 38X higher than before the pandemic, and industry analyst, IDC (International Data Corporation), 
also predicts that by 2023, nearly two-thirds of patients will have accessed healthcare via a digital front end (2). Factors for growth in adoption include favorable consumer perception, improved regulatory environment, and strong investment into this space.

What does this mean for the pharma industry?

With blockchain maturing even more in the pharma supply chain, the promise of a single system accessible to all parties involved in a supply chain transaction, and by design immutable, makes blockchain supply chain traceability even more important in a virtual transaction from plant to patient.

The aims are quite simple - enable greater traceability, transparency, and the automation of commercial processes and maybe even become an acceptable form of payment for supply chain products and services.

Traceability and transparency reflect the market's need for a single source of truth in the supply chain - from a raw material's provenance to a product's end of life. A recent industry survey concluded that product traceability throughout production and the supply chain is the primary use case for blockchain-adopting industry giants across multiple industries.

As for automating commercial processes through blockchain-based smart contracts, by doing this, we can hugely streamline service and payment transactions while reducing errors in the back office. When predetermined service level agreement criteria are met, smart contracts allow procedures to self-execute without relying on human participation.

Given the abovementioned factors for growth in the telehealth industry, we foresee them having positive effects on the adoption of blockchain for the pharma industry as well, helping to ensure safe and more accessible healthcare, overall.

Finally, will we see cryptocurrency make its way into the mainstream as an alternate form of payment for telehealth services? While volatility is still a concern, just like the debit, credit, and methods of payment that precede them, cryptocurrencies are a viable payment method for logistics products and services, and supplementary acceptance may strengthen logistics providers' competitiveness and further their integration in the digital consumer world.

\section{Gabriela Pelin, Chief Innovation Officer, Avaneer Health, USA}

Over the past few years, an increasing number of healthcare organizations have begun to evaluate and adopt blockchain-based solutions. The technological landscape is changing from technology assisting independently run processes to being capable of running peer-to-peer interactions and joint processes. In 2022, healthcare companies will begin looking beyond 'better app' to seek better connectivity and business process scaling to coordinate care delivery and administration that creates direct interactions over digital highways. Implementing and using blockchain will require a new strategy for payers and providers - one based on a new mindset of coopetition.

While embracing this new mindset may be a big hurdle to overcome, we all agree that the fluidity of data exchange between patients, providers, payers, and vendors is essential for delivering quality care and an optimal patient experience. This is where blockchain shines and will make an impact on the healthcare experience in this decade. Decentralized ledgers that hold accurate and immutable information about the information and prove the provenance of data are essential for doing business. In short, blockchain is a catalyst for making true interoperability possible.

To bring about blockchain's full potential for healthcare, however, we need a comprehensive network equipped with FHIR (Fast Healthcare Interoperability Resources) servers, where all stakeholders can commit data to be discoverable based on the parameters they set. Network users will connect to the network via the cloud, where an ID keychain and master index locate the information requested and match it to the data available, then deliver it to the requestor. A network intermediary will provide certification, cybersecurity, and compliance. Having this central blockchain network eliminates the need for organizations to build and maintain multiple gateways with multiple entities. This significantly reduces IT costs and enables more organizations to participate in all the benefits blockchain has to offer.

\section{Anton Hesselgren, Department of Neuromedicine and Movement Science, Faculty of Medicine and Health Sciences (NTNU), Norwegian University of Science and Technology, Trondheim, Norway}

The year 2021 has been exciting for blockchain technology. Cryptocurrencies and blockchain decentralized platforms have taken a leap forward, and the use-cases in healthcare have seen an increased focus, particularly due to the COVID-19 pandemic. It has become prominent that decentralized technologies that enable trust between entities, where trust is lacking, are needed in the healthcare space. Again, the pandemic has shown us that there is a lack of trust in public health and healthcare.

For the year 2022, first, I believe we will have more developed and robust vaccination certificate solutions built on blockchains, not just for COVID-19 but for all vaccinations. These solutions could scale and also work as means to prove other types of delivered healthcare services. Second, I believe that blockchain will be an important component in clinical trial management with transparent and tamper-proof informed consent management, in particular in decentralized clinical trials. These will benefit greatly by a decentralized data structure that also can provide data provenance and tamper-proof data. During the coming year, we will see more solutions in this area ready for implementation. 


\section{Conclusion: Daniel Conway}

While we will know more in 2023, a quick look at the predictions above should generate great excitement. These are not fringe nice-to-have innovations being described-, but rather visions that will impact the core value offering of healthcare itself. Many will involve further ethical discussion regarding control of information.

Ultimately, blockchains provide transparency and a substitute form of trust. They enable efficiencies in the health ecosystem, which were never feasible in the past. These are essential characteristics of any healthcare system. Blockchain does not have a mechanism that forgets or can be amended. Immutability has different characteristics than traditional records systems controlled by individual organizations. This suggests new thinking in organizational controls and collaboration. It will certainly be an interesting year.

\section{Funding Statement}

The authors have not received any funding or benefits from industry or elsewhere to conduct this study.

\section{Financial and non-Financial Relationship and Activities}

Daniel Laverick is a current employee of Zuellig Pharma Holdings Pte Ltd, which has not sponsored, funded, or endorsed this work.

\section{Authors' contributions}

All authors contributed substantially to the research, including study design, data management, data analysis, data interpretation, and manuscript preparation.

\section{References}

1. Bestsennyy O, Gilbert G, Harris A, Rost J. Telehealth: A quarter-trillion-dollar post-COVID-19 reality? [Internet]. McKinsey \& Company; 2021 [updated 2021]. Available from: https:// www.mckinsey.com/industries/healthcare-systems-and-services/ our-insights/telehealth-a-quarter-trillion-dollar-post-covid-19reality [cited 28 October 2021].

2. Miliard M. What to expect in 2021 and beyond? IDC offers 10 healthcare predictions [Internet]. Healthcare IT News; 2020 [updated 2020]. Available from: https://www.healthcareitnews.com/ news/what-expect-2021-and-beyond-idc-offers-10-healthcarepredictions [cited 28 October 2021].

Copyright Ownership: This is an open access article distributed in accordance with the Creative Commons Attribution Non Commercial (CC BY-NC 4.0) license, which permits others to distribute, adapt, enhance this work non-commercially, and license their derivative works on different terms, provided the original work is properly cited and the use is non-commercial. See: http://creativecommons. org/licenses/by-nc/4.0. 